\title{
Towards a Standard Experimental Protocol for Open Source Additive Manufacturing
}

\begin{abstract}
The technological development of open source 3D printers is creating more affordable Additive Manufacturing (AM) machines for society in different applications. For this reason, the machines' capability should be evaluated in order to establish minimum standards of performance. This paper deals with the development, manufacture and testing of a geometrical benchmarking model (GBM) in order to evaluate the geometrical accuracy performance of open source 3D printers. The methodology is demonstrated with a case study based on fused deposition modeling (FDM). The case study positions the evaluated machine according to ANSI-ISO's International Standard tolerance grade (IT). Furthermore, root-meansquare deviation (RMSD) value is employed as an accuracy estimator, while Taguchi tools are employed to determinate the control factors with the highest accuracy for the fabrication of the GBM.
\end{abstract}

Keywords: Open Source 3-Dimensional printing, Machine qualification, RepRap, Benchmarking.

\section{Introduction}

The convergence of information and communication technologies (ICT) with digital fabrication capabilities of Additive Manufacturing (AM), specifically the development of open source 3D printers, is creating the appropriate knowledge-based social environments that enable independent production of modular hardware. This synergy could be transformed into a new disruptive paradigm of means of production for modular hardware (Kostakis \& Papachristou 2014). In particular, material extrusion based units are widely used, thanks to the simplicity of operation, the Do-It-Yourself (DIY) approach and the open-support communities. It provides the possibility of mass diffusion of this technology, and consequently, AM is being recognised as a revolutionary technology that could up-end the last two centuries of approaches to design and manufacturing with profound geopolitical, economic, social, demographic, environmental and security implications (Pearce 2014; Birtchnell \& Urry 2013; Garrido 2010; Campbell et al. 2011; Rifkin 2012; Economist 2012)

With the expiration of Fused Deposition Modelling (FDM) patents (Crump 1988) in the mid-2000s, Adrian Bowyer envisioned the concept of self-replicating machines, capable of manufacturing their own parts by themselves, and so simple and easy that anyone would be able to build them (Holland, D, O'Donnell, G., Bennett 2010; Sells et al. 2007; Jones et al. 2011). This was the start of the RepRap project (or Replicating Rapid-prototyper). RepRap is a low-cost desktop rapid prototyper which manufactures approximately $57 \%$ of its own mechanical components (excluding fasteners, bolts and nuts). This project has been developed using an Open Design approach in which detailed information on the technical design and operations of the device is publicly available on the internet. In the literature, RepRaps have been proved to be useful tools in fields such as transport (Birtchnell \& Urry 2013), education (Canessa, Fonda, \& Zennaron 2013), engineering (Kostakis \& Papachristou 2014; Sells et al. 2007), tissue engineering (Ciurana, Serenóa, \& Vallès 2013), chemical reaction wire, customising scientific equipment (Zhang et al. 2013; Pearce 2012; Ter 2014), electronic sensors (Leigh et al. 2012), wire embedding (Bayless, Chen, \& Dai 2010) and appropriate technology related for sustainable development (Pearce et al. 2010).

Characteristics of the RepRap project, such as its open source nature and its customisation and selfreplication capability, open up the possibility for exponential growth for both products and 3D printer systems. The RepRap project has been an object of social experimentation, creating numerous enthusiasts and communities interested in supporting various RepRap models. Different parallel open source systems 
[Pre-print version], please cite as: Cruz Sanchez, F. A., Boudaoud, H., Muller, L., \& Camargo, M. (2014). Towards a standard experimental protocol for open source additive manufacturing. Virtual and Physical Prototyping, 9(3), 151-167

have emerged, such as Fab@Home 3D printer (Malone \& Lipson 2007), the CupCake CNC and Thing-O-Matic 3D printers by MakerBot Inc (MakerBot 2014) and others. The RepRap website invites machine developers to register their project in a database in order to collect the total of different prototypes and projects. According to this database, there are approximately 500 models (RepRap.org 2014). This exponential growth makes it essential to evaluate the capabilities of machines in order to characterize and differentiate them. In fact, attention has been drawn to the relevance of logical evaluation tools for individuals to allow a fair comparison of the performance of a given unit to another through the use of a benchmarking process (Roberson, Espalin, \& Wicker 2013; Perez et al. 2013).

In the context of commercial Additive Manufacturing, several benchmarking propositions have been made to evaluate the performance of the techniques. Mahesh et al. (2006) present a methodology for identifying the best achievable quality characteristics to serve as a benchmark process using a case study involving the DLS process. Scaravetti, Dubois, \& Duchamp (2007) worked on a benchmarking model and a procedure using a correlation matrix, in order to identify the defects of SL processes for establishing whether their origins are machine or material linked. In the case of open source Additive Manufacturing, Johnson et al. (2011) have evaluated an open source AM system based on FDM technique through use of a benchmarking model in order to assess the dimensional accuracy, feature size and geometry limitation, geometric and dimensional tolerance. The fabricated model was evaluated using a 3D laser scanning system, which has an accuracy of $0.0089 \mathrm{~mm}$ far below than the minimum feature size limitation of the used printer $(0.08 \mathrm{~mm})$. Using nearly 3 million of data point, they observed a standard deviation of $0.3101 \mathrm{~mm}$ between the fabricated model and the CAD model, with $98.146 \%$ of the points within $+/-2$ standard deviations.

Roberson, Espalin, \& Wicker (2013) propose a framework to evaluate the performance of a set of five 3D printer units, establishing a ranking method based on four factors (manufacturing time, cost of machine, material cost and dimensional accuracy) in order to establish a hierarchy among them. However, the authors recognised that a robust evaluation and comparison of the various AM systems are required and this research is still at an early stage. Indeed, it remains to be demonstrated that open-source systems are capable of making objects in a robust way with good reproducibility. This evaluation, from the AM system user's point of view, will make it possible to know the performance of the different open source systems. On the other hand, from the point of view of an AM system developer, the evaluation of the reproducibility will enable to determine optimal parameters of the process in order to replicate the system, ensuring the evolution of performance from generation to generation of the machines.

Following this path of research, this paper proposes an experimental protocol of geometrical performance evaluation in order to characterise open source 3D printers in a robust manner. Based on Design of Experiments (DoE) and integrating different families of geometrical objects, the dimensional accuracy of a representative 3D printer is intensively studied.

This paper is structured as follows: in section 2 an overview of the methodology is proposed. Subsequently, in section 3, the proposed methodology is applied in the case of the open source 3D printer FoldaRap. The results and the discussion are shown in section 4 and concluding remarks and perspective are given in section 5 .

\section{Methodology}

There are two goals in the deployment of the methodology. The first is to evaluate the performance of an open source AM machine in terms of dimensional accuracy and reproducibility through statistical analysis of the set of manufactured samples. This quantitative qualification will make it possible to establish a characterisation of machine performance, in terms of four types of dimensional accuracy, as follows:

- XY- Plane

- Z-Axis.

- Circular features -D-

- Thin walls - T-

Once the degree of reproducibility of the machine is verified, the second goal is to find the parameters of the 3D printer machine among the parameters tested that give the lowest dimensional accuracy discrepancies 
[Pre-print version], please cite as: Cruz Sanchez, F. A., Boudaoud, H., Muller, L., \& Camargo, M. (2014). Towards a standard experimental protocol for open source additive manufacturing. Virtual and Physical Prototyping, 9(3), 151-167

possible for the fabrication of a benchmarking model. Fig. 1 shows an overview of the different steps of the proposed methodology. These steps will be detailed as follows:

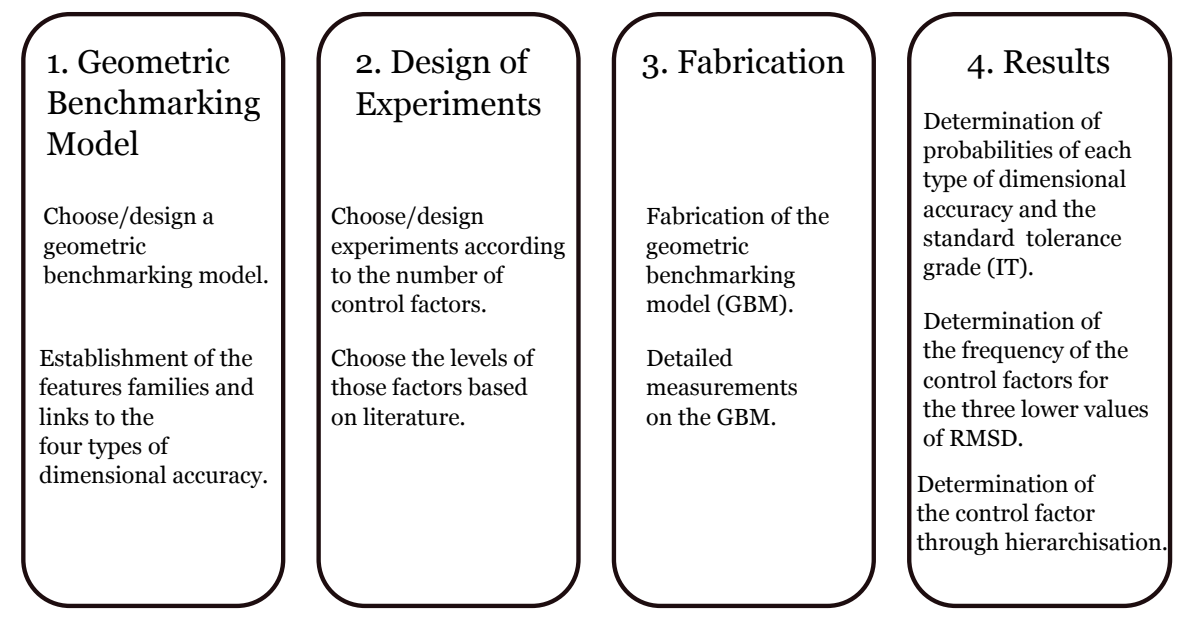

Figure 1: Overview of the proposed methodology

\subsection{Geometric Benchmarking Model}

Benchmarking is a tool for comparing the performance of different similar systems (processes, organisations, machines) in order to establish standards of performance. It aims to identify the best achievable practices and processes. In this step, the goal is to identify a model of reference intended for the evaluation of the open source AM system. Once a benchmarking model is adopted, it is intended to link different types of feature families with the four types of dimensional accuracies proposed.

In the commercial AM field, there have been several efforts to perform benchmarking studies for different processes through the use of comparative models. Kruth (1991) was the first to mention a benchmarking part for comparing AM processes, citing a study done by two Dutch companies using a U-shaped artefact with various geometric features such as circular shells (in various orientations), circular bosses, square holes and angled surfaces. This benchmark part focuses on the overall performance of the AM systems. Ippolito, Iuliano, \& Gatto (1995) worked on the development, manufacture and test of a benchmarking model in order to investigate dimensional accuracy and surface finish of various AM techniques such as SL, SLS, FDM, LOM and SGC. Jayaram et al. (1994) aim to lay the groundwork for the development of standards to measure various performance factors such as repeatability, warpage, curl, creep, shrinkage and tensile strength in a quantitative way; test parts were designed for studying SL, SLS, LOM and FDM techniques. In 2000, Xu, Wong, \& Loh (2000) presented a benchmarking model to evaluate differences on the material property, accuracy, surface finish, building cost-time, machinability and environmental effects of the SL, SLS, FDM and LOM processes. Mahesh et al. (2006) proposed a benchmarking model using a Six-sigma approach in order to (1) minimise process inconsistencies and defects of fabrication, and (2), to identify a best process/procedure to achieve desirable geometric accuracy and surface roughness in the DLS process. Fahad \& Hopkinson (2012) proposed a geometric benchmarking part for evaluating the accuracy, tolerances and repeatability of parts produced by different AM processes. In summary, Mahesh (2004) identifies three types of benchmarking models in AM, as follows:

Geometric Benchmark; used to check the geometric and dimensional accuracy of the prototype (i.e. tolerances, accuracy, repeatability and surface finish).

Mechanical Benchmark; used to characterise the mechanical properties (i.e. tensile/compression strength, shrinkage, curling and creep characteristics.)

Process Benchmark; used to establish process related parameters (part orientation, support structures, layer thickness, speed) 
[Pre-print version], please cite as: Cruz Sanchez, F. A., Boudaoud, H., Muller, L., \& Camargo, M. (2014). Towards a standard experimental protocol for open source additive manufacturing. Virtual and Physical Prototyping, 9(3), 151-167

In addition, Moylan et al. (2012) summarise some items to consider in order to establish 'rules' for a geometric benchmarking model. Globally, benchmarking models should:

- be large enough to test the performance of the machine near the edges of the platform as well as near the centre,

- have a substantial number of small, medium and large features,

- not take long to build,

- not consume a large quantity of material,

- be easy to measure,

- have many features of a 'real' part,

- have simple geometrical shapes, allowing perfect definition and easy control of the geometry,

- require no post-treatment or manual intervention (No support structures),

- allow repeatability measurements,

Based in this literature, the objective of this step is to reach a final design of a Geometric Benchmarking Model (GBM). This design should incorporate geometric shapes and features that provide important information of the capabilities and limitations of the open source 3D printer analysed.

\subsection{Design of Experiments}

The goal of this step is to establish the control factors to evaluate, the fixed factors to consider, and the sequence and quantity of samples to manufacture. One of the available approaches is the Taguchi method, which has been proven to be successful for improvement of product quality and process performance. This method provides an efficient and systematic approach to optimize a number of experiments and the feasibility of studying of interaction effects among parameters, while maintaining valid conclusions (Azadeh et al. 2011; Fowlkes \& Creveling 1995). In the literature of commercial AM, there have been several attempts to improve the dimensional accuracy of prototypes using adjustment of the process parameters. Using Taguchi method, Zhou, Herscovici, \& Chen (2000) worked on the accuracy of rapid prototyped SL parts analysing five factors, namely layer thickness, hatch spacing, overcure, blade gap and position on the build plane. They used ANOVA approach to develop a second-order regression model establishing the best parameters to reduce the dimensional error to the smallest value. Anitha, Arunachalam, \& Radhakrishnan (2001) assessed the influence of the layer thickness, road width and speed deposition on the surface roughness of the prototypes produced by the FDM process in order to minimise the surface roughness. The results indicate that layer thickness is the most influential process parameter affecting roughness, followed by road width and deposition speed. Mahesh et al. (2006) used the Taguchi method on the Direct Laser Sintering -DLS- process in order to determine the setting of the key control factors for obtaining the best achievable result in terms of geometric accuracy and surface roughness for different individual geometric features such as sphere circle, cone, cylinders, square boss and wedge. Sood, Ohdar, \& Mahapatra (2009) investigated the effects of process parameters (orientation, layer thickness, raster angle, raster width and air gap) on FDM dimensional accuracy in order to reduce the percentage change in length, width and thickness of a test specimen. Results show shrinkage is dominant along the length and width of the test part, whereas thickness is always more than the desired value.

The authors are persuaded to adopt this method to evaluate the performance of an AM system in order to characterise the performance of the 3D printer.

\subsection{Fabrication and Measurement of Geometric Benchmarking Model}

The fabrication of the GBM is performed according to the instructions of the experiment design. It is intended in this protocol to use AM systems of an open source nature (less than US\$1000). The selection of the machine to evaluate should be representative among the ensemble of AM systems currently in existence.

\subsection{Results Accuracy Index and Statistical Analysis}

The goal of this step is to quantify the dimensional accuracy of the machine, establishing a range of tolerance and confidence interval of the machine for every kind of dimensional accuracy proposed and to quantify the 
[Pre-print version], please cite as: Cruz Sanchez, F. A., Boudaoud, H., Muller, L., \& Camargo, M. (2014). Towards a standard experimental protocol for open source additive manufacturing. Virtual and Physical Prototyping, 9(3), 151-167

probability of reproducibility of the machine inside a range of deviation. The second goal is to establish an accuracy index for the control factors, which will make it possible to rank the set of combinations of control factors in order to determinate those with the highest dimensional accuracy. In the next section, the approach will be illustrated by means of the evaluation of an open source AM system.

\section{Application to an OS AM System: the Case of the FoldaRap}

\subsection{Equipment}

A derivative version of the RepRap machine, called FoldaRap (Gilloz 2014) (see fig. 2) was selected for this investigation. It is the first open source 3D printer designed to fold into a very small size to be completely portable. It is an open-source design and it comes from a derivation of eMAKER Huxley (Giacalone 2014), VertX (Gilloz 2011b) and Pocket Laser Engraver (Gilloz 2011a). The FoldaRap machine is a representative 3D printer among the set of open source machines developed by the RepRap community. Indeed, as can be seen in the open source 3D printer family tree (Pearce 2014), the FoldaRap derives from the main branch (XZ Head, Y Bed): Darwin-Sells Mendel-Prusa Mendel.

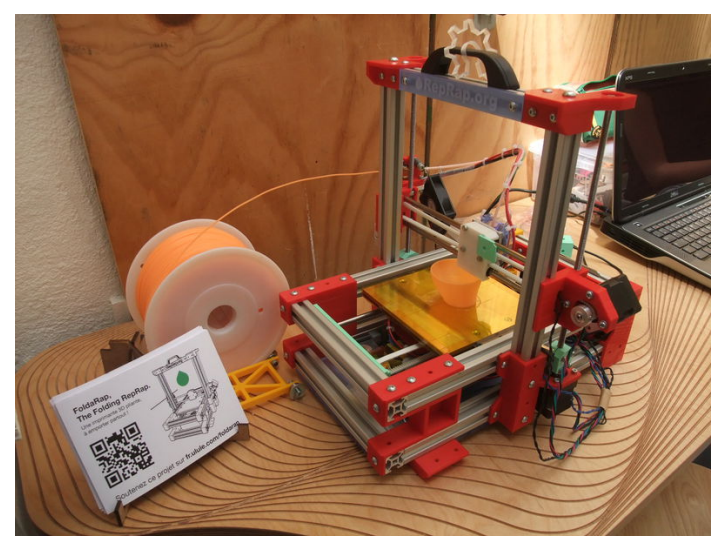

Figure 2: Open Source 3D printer -FoldaRap-

This system can be described through three fundamental axes (Evans 2012; RepRapWiki 2014):

1. Machine architecture

2. Electronic hardware

3. Software

Regarding the FoldaRap's architecture, it is a Cartesian 3D printer where the extrusion system can be displaced in the vertical plane XZ and the heated print bed can be displaced in the horizontal direction -Y. The work capability is 140X140X155 $\mathrm{mm}^{3}$. Using a mechanical coupling stepper motor-drive gear, the extrusion system forces a PolyLactic Acid (PLA) polymer filament with a diameter of $1.75 \mathrm{~mm}$ into an aluminium melt chamber, then the filament is extruded through a $0.5 \mathrm{~mm}$ nozzle. The linear motion for positioning axis $\mathrm{XY}$ is achieved through machined plastic bushings and smooth rods $6 \mathrm{~mm}$ in diameter using a transmission mechanism of timing belts and pulleys. For axis Z, threaded rods M5 and hexagonal nuts are coupled with a stepper motor with a minimum resolution of $0.00025 \mathrm{~mm}$. The heated print bed is made of aluminium joined with a Peltier cell and it uses a top layer of kapton in order to improve the adherence of the piece with the print bed.

Concerning the electronic hardware and software, the FoldaRap machine uses a Melzi v2.0 controller board which makes it possible to control the machine via a USB connection (RepRapPro 2014) . Marlin is used as firmware software, Slic3r software is used to convert the .STL files into G-codes, and Pronterface software is used as the system's host software. All these elements are open source.

Foldarap has an indicated minimum value of layer height tested at $0.1 \mathrm{~mm}$ and it does not include the capacity to fabricate support structures. Figure 3 shows the structure of the machine schematically. 
[Pre-print version], please cite as: Cruz Sanchez, F. A., Boudaoud, H., Muller, L., \& Camargo, M. (2014). Towards a standard experimental protocol for open source additive manufacturing. Virtual and Physical Prototyping, 9(3), 151-167
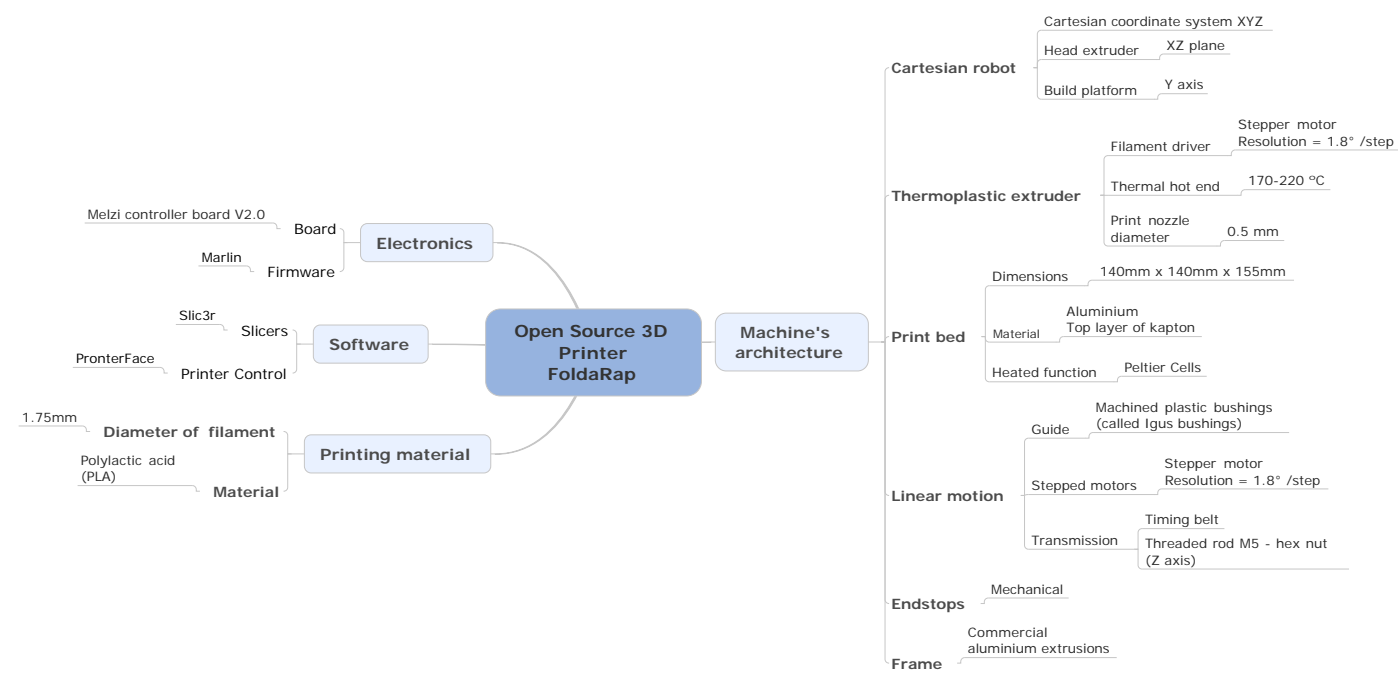

Figure 3: Main components of the FoldaRap 3D printer

\subsection{Benchmarking Models in Additive Manufacturing}

Based on the discussion of section 2.1, this experiment uses a modified version of the model of the National Institute of Standards and Technology (NIST) (Moylan et al. 2012). The model is shown in figure 4 and table 1 summarises the features and characteristics. The modifications made in the benchmarking models have been in order to link different types of features with different types of accuracies of the machine proposed in this investigation.

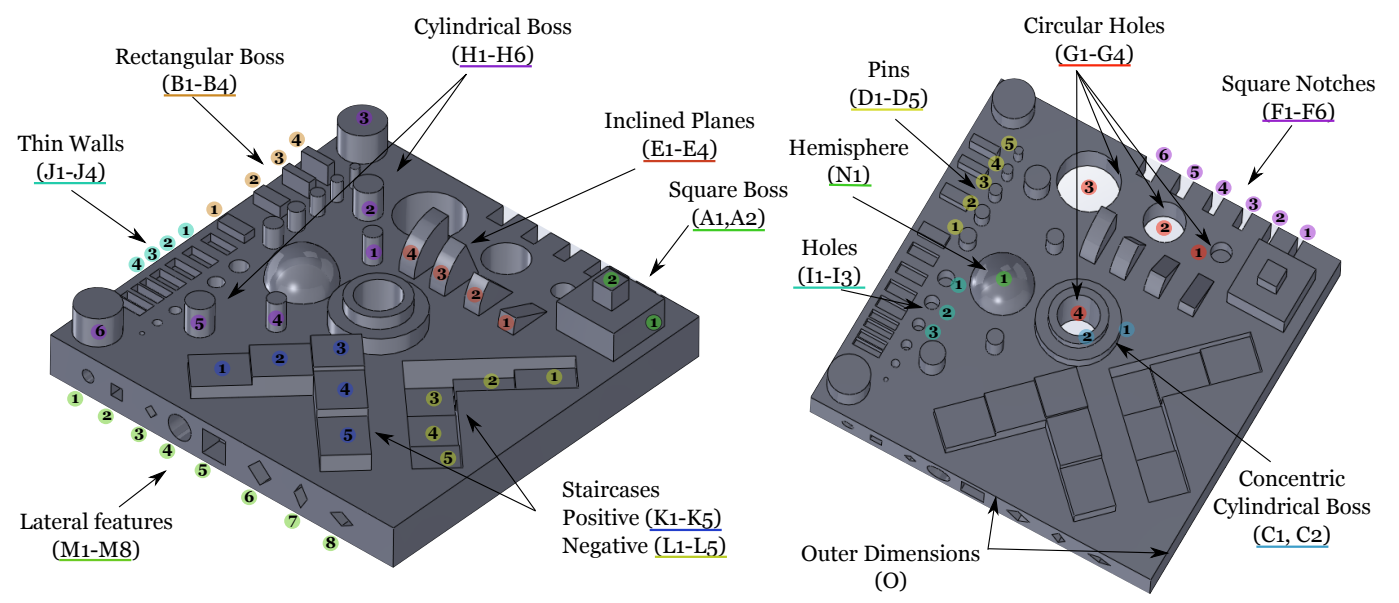

Figure 4: CAD version of benchmarking model with referenced feature identification (IDs)

The geometric benchmarking model is divided into 15 different types of family groups. Each family group is formed by various related features where each feature is systematically identified with a letter (A-O) and a number. Every feature is associated with at least one type of dimensional accuracy with the objective of evaluating each type of dimensional accuracy proposed. The dimensional accuracy can therefore be analysed 
[Pre-print version], please cite as: Cruz Sanchez, F. A., Boudaoud, H., Muller, L., \& Camargo, M. (2014). Towards a standard experimental protocol for open source additive manufacturing. Virtual and Physical Prototyping, 9(3), 151-167

with different features of the model; in this way, the dimensional accuracy obtained comes from a set of features. This approach will make it possible to characterize the performance of the open source 3D printer, and consequently it will make it possible to differentiate the machine from the other open source machines.

Table 1: Benchmarking model feature descriptions

\begin{tabular}{|c|c|c|c|}
\hline ID & $\begin{array}{l}\text { Family of } \\
\text { features }\end{array}$ & Features & Description \\
\hline A & Square Boss & A1-A2 & $\begin{array}{l}\mathrm{A} 1=15 \times 15 \times 17 \mathrm{~mm} \\
\mathrm{~A} 2=5 \times 5 \times 22 \mathrm{~mm}\end{array}$ \\
\hline B & $\begin{array}{l}\text { Rectangular } \\
\text { Boss }\end{array}$ & B1-B4 & $\mathrm{B} 1=7 \times 3 \mathrm{X} 12 \mathrm{~mm}, \mathrm{~B} 2=7 \times 3 \times 13 \mathrm{~mm}$ \\
\hline $\mathrm{C}$ & $\begin{array}{l}\text { Concentric } \\
\text { cylindrical } \\
\text { boss }\end{array}$ & $\mathrm{C} 1-\mathrm{C} 2$ & $\begin{array}{l}\mathrm{B} 3=7 \times 2 \times 14 \mathrm{~mm}, \mathrm{~B} 4=7 \times 2 \times 15 \mathrm{~mm} \\
\mathrm{C} 1=\varnothing 20 \mathrm{~mm}\end{array}$ \\
\hline D & Pins & D1-D5 & $\begin{array}{l}\mathrm{C} 2=\varnothing 14 \mathrm{~mm} \\
\mathrm{D} 1=\varnothing 4 \mathrm{~mm}, \mathrm{D} 2=\varnothing 3.5 \mathrm{~mm}, \mathrm{D} 3=\varnothing 3 \mathrm{~mm}, \\
\mathrm{D} 4=\varnothing 2.5 \mathrm{~mm} \& \mathrm{D} 5=\varnothing 2 \mathrm{~mm} .\end{array}$ \\
\hline $\mathbf{E}$ & Inclines & E1-E4 & $\begin{array}{l}\mathrm{E} 1=13.83 \mathrm{~mm}\left(15^{\mathrm{o}}\right), \mathrm{E} 2=17.07 \mathrm{~mm}\left(45^{\mathrm{o}}\right) \\
\mathrm{E} 3=19.24 \mathrm{~mm}\left(75^{\mathrm{o}}\right), \mathrm{E} 4=20 \mathrm{~mm}\left(90^{\mathrm{o}}\right) .\end{array}$ \\
\hline $\mathbf{F}$ & $\begin{array}{l}\text { Square } \\
\text { Notches }\end{array}$ & F1-F6 & $\mathrm{F} 1=1.5 \mathrm{~mm}, \mathrm{~F} 2=2 \mathrm{~mm}, \mathrm{~F} 3=2.5 \mathrm{~mm}$ \\
\hline G & $\begin{array}{l}\text { Circular } \\
\text { Holes }\end{array}$ & G1-G4 & $\begin{array}{l}\mathrm{F} 4=3 \mathrm{~mm}, \mathrm{~F} 5=3.5 \mathrm{~mm}, \mathrm{~F} 6=4 \mathrm{~mm}, \\
\mathrm{G} 1=\varnothing 5 \mathrm{~mm} . \text { (Prof. } 5 \mathrm{~mm} \text { ) }\end{array}$ \\
\hline & & & $\begin{array}{l}\mathrm{G} 2=\varnothing 10 \mathrm{~mm}, \mathrm{G} 3=\varnothing 15 \mathrm{~mm}, \\
\mathrm{G} 4=\varnothing 10 \mathrm{~mm}\end{array}$ \\
\hline $\mathbf{H}$ & $\begin{array}{l}\text { Cylindrical } \\
\text { Boss }\end{array}$ & H1-H6 & $\mathrm{H} 1, \mathrm{H} 4=\varnothing 4 \mathrm{~mm}$ \\
\hline & & & $\begin{array}{l}\mathrm{H} 2, \mathrm{H} 5=\varnothing 6 \mathrm{~mm} \\
\mathrm{H} 3, \mathrm{H} 6=\varnothing 10 \mathrm{~mm}\end{array}$ \\
\hline $\mathbf{I}$ & Holes & I1-I3 & $\begin{array}{l}\mathrm{I} 1=\varnothing 4 \mathrm{~mm}, \mathrm{I} 2=\varnothing 3.5 \mathrm{~mm}, \mathrm{I} 3=\varnothing 3 \mathrm{~mm} \\
\text { Prof. } 5 \mathrm{~mm} .\end{array}$ \\
\hline $\mathbf{J}$ & Thin walls & J1-J4 & $\begin{array}{l}\mathrm{J} 1=2 \mathrm{~mm}, \mathrm{~J} 2=1.5 \mathrm{~mm} \\
\mathrm{~J} 3=3 \mathrm{~mm}, \mathrm{~J} 4=3 \mathrm{~mm}\end{array}$ \\
\hline K & $\begin{array}{l}\text { Positive } \\
\text { Staircases }\end{array}$ & K1-K5 & Height $2,4,5,6, \& 7 \mathrm{~mm}$ \\
\hline $\mathbf{L}$ & $\begin{array}{l}\text { Negative } \\
\text { Staircases }\end{array}$ & L1-L5 & $\begin{array}{l}\text { width } 10 \mathrm{~mm} \text {. } \\
\text { Height } 2,4,5,6 \& 7 \mathrm{~mm} .\end{array}$ \\
\hline M & $\begin{array}{l}\text { Lateral Fea- } \\
\text { tures }\end{array}$ & M1-M8 & $\begin{array}{l}\text { width } 10 \mathrm{~mm} . \\
\mathrm{M} 1=\varnothing 3 \mathrm{~mm}, \mathrm{M} 2=3 \times 3 \mathrm{~mm}, \mathrm{M} 3=3 \times 3 \mathrm{~mm} \text {, }\end{array}$ \\
\hline $\mathbf{N}$ & Hemisphere & N1 & $\begin{array}{l}\mathrm{M} 4=\varnothing 6 \mathrm{~mm}, \mathrm{M} 5=6 \times 6 \mathrm{~mm}, \mathrm{M} 6=6 \times 6 \mathrm{~mm} \\
\mathrm{M} 7=6 \mathrm{~mm}(\mathrm{Z} \text {-axis }), \mathrm{M} 8=6 \mathrm{~mm} . \\
\text { Features in the vertical plane } . \\
\mathrm{r}=8 \mathrm{~mm} .\end{array}$ \\
\hline $\mathrm{O}$ & $\begin{array}{l}\text { Outer } \\
\text { dimensions }\end{array}$ & $\mathrm{X}-\mathrm{Y}-\mathrm{Z}$ & $90 \times 90 X 10 \mathrm{~mm}$ \\
\hline
\end{tabular}


[Pre-print version], please cite as: Cruz Sanchez, F. A., Boudaoud, H., Muller, L., \& Camargo, M. (2014). Towards a standard experimental protocol for open source additive manufacturing. Virtual and Physical Prototyping, 9(3), 151-167

Table 2: Corresponding geometric features of each type of dimensional accuracy

\begin{tabular}{|c|c|c|}
\hline $\begin{array}{l}\text { Dimensional } \\
\text { Accuracy }\end{array}$ & Features & \\
\hline XY Plane & $\begin{array}{l}\mathrm{A} 1, \mathrm{~A} 2 \\
\mathrm{~B} 1-\mathrm{B} 4 \\
\text { K1-K5 } \\
\text { L1-L5 } \\
\text { M2,M3,M5,M6,M8 } \\
\mathrm{O}\end{array}$ & $\begin{array}{l}\text { \# of measures } \\
\text { per sample } \\
\mathrm{X}=22 \\
\mathrm{Y}=17\end{array}$ \\
\hline Z- Direction & $\begin{array}{l}\mathrm{A} 1, \mathrm{~A} 2 \\
\mathrm{~B} 1-\mathrm{B} 4 \\
\text { E1-E4 } \\
\text { K1-K5 } \\
\text { L1-L5 } \\
\text { M2,M3,M5,M6,M7 } \\
\text { N1 } \\
\mathrm{O}\end{array}$ & \\
\hline Diameters & $\begin{array}{l}\text { C1,C2 } \\
\text { D1-D5 } \\
\text { G1-G4 } \\
\text { H1-H6 } \\
\text { I1-I3 } \\
\text { M1,M4 }\end{array}$ & \\
\hline Thin walls & $\begin{array}{l}\text { F1-F6 } \\
\text { J1-J4 }\end{array}$ & \\
\hline
\end{tabular}


[Pre-print version], please cite as: Cruz Sanchez, F. A., Boudaoud, H., Muller, L., \& Camargo, M. (2014). Towards a standard experimental protocol for open source additive manufacturing. Virtual and Physical Prototyping, 9(3), 151-167

\subsection{Design of Experiments}

Based on the literature described in section 2.2, three control factors have been considered in this investigation. They are briefly defined as follows (Kumar Sahu 2011; Cantini \& Bortolin 2012).

- Layer thickness: The thickness of a layer deposited by the nozzle, which depends upon the type of nozzle used.

- Extrusion width: The value of the width of the filament that leaves the printer nozzle.

- Nozzle speed: The speed of the printer nozzle when it fabricates the object. (Speed of perimeters, small perimeters, external perimeters, infill -solid, top, bottom layers -).

Table 3 shows the respective levels of each factor.

Table 3: Control factors

\begin{tabular}{lccccc}
\hline & \multicolumn{5}{c}{ Levels } \\
\cline { 3 - 5 } Control factors & ID & $\mathbf{1}$ & $\mathbf{2}$ & $\mathbf{3}$ & Units \\
\hline & & & & & \\
Layer thickness & $\mathrm{A}$ & 0.13 & 0.18 & 0.25 & $\mathrm{~mm}$ \\
Raster width & $\mathrm{B}$ & 0.54 & 0.62 & 0.71 & $\mathrm{~mm}$ \\
Nozzle speed & $\mathrm{C}$ & 25 & 50 & 75 & $\mathrm{~mm} / \mathrm{s}$ \\
\hline
\end{tabular}

On the other hand, fixed factors considered in this investigation are shown in table 4.

Table 4: Fixed factors

\begin{tabular}{lcc}
\hline Parameters & Value & Units \\
\hline Bed temperature & 52 & ${ }^{\circ} \mathrm{C}$ \\
Nozzle temperature & 190 & ${ }^{\circ} \mathrm{C}$ \\
\# of perimeters & 2 & \\
Top solid layers & 2 & \\
Bottom solid layers & 2 & \\
Fill density & 30 & $\%$ \\
Material & PLA & \\
\# of repetitions & 2 & \\
Travel speed & 200 & $\mathrm{~mm} / \mathrm{s}$ \\
Nozzle diameter & 0.5 & $\mathrm{~mm}$ \\
Filament Diameter & 1.75 & $\mathrm{~mm}$ \\
Support & Non & \\
Slic3r software for gen- & & \\
eration of G-code & & \\
\hline
\end{tabular}

In classical Design of Experiments (DOE), the study of three factors at three levels would require $27\left(3^{3}\right)$ experiments. Using Taguchi's approach, a reliable estimation of the effect of factors can be obtained by using an orthogonal array with fewer experiments. The appropriate orthogonal array for this experiment is $L_{9}\left(3^{4}\right)$. This array consists of nine rows for the experiment conditions of the control factors and four columns for assigning the factors or interactions. Columns 1,2 and 4 are used for control factors A, B and C respectively. Table 5 shows the $L_{9}$ orthogonal array used in this investigation. 
[Pre-print version], please cite as: Cruz Sanchez, F. A., Boudaoud, H., Muller, L., \& Camargo, M. (2014). Towards a standard experimental protocol for open source additive manufacturing. Virtual and Physical Prototyping, 9(3), 151-167

\begin{tabular}{|c|c|c|c|}
\hline \multirow[t]{2}{*}{ \# Sample } & \multicolumn{3}{|c|}{ Control factors } \\
\hline & A & B & $\mathrm{C}$ \\
\hline 1 & 1 & 1 & 1 \\
\hline 2 & 1 & 2 & 2 \\
\hline 3 & 1 & 3 & 3 \\
\hline 4 & 2 & 1 & 3 \\
\hline 5 & 2 & 2 & 1 \\
\hline 6 & 2 & 3 & 2 \\
\hline 7 & 3 & 1 & 2 \\
\hline 8 & 3 & 2 & 3 \\
\hline 9 & 3 & 3 & 1 \\
\hline
\end{tabular}

\subsection{Fabrication}

Using Taguchi's array proposed in the previous section, and the geometric benchmarking model developed in section 3.2, the open source 3D printer 'FoldaRap' manufactured a total of eighteen samples. That means that two samples were fabricated for each row of the array. Fig. 5 shows the coordinates system used by the $3 \mathrm{D}$ printer for manufacturing the samples. It is $45^{\circ}$ with respect to the outer edges of the model.

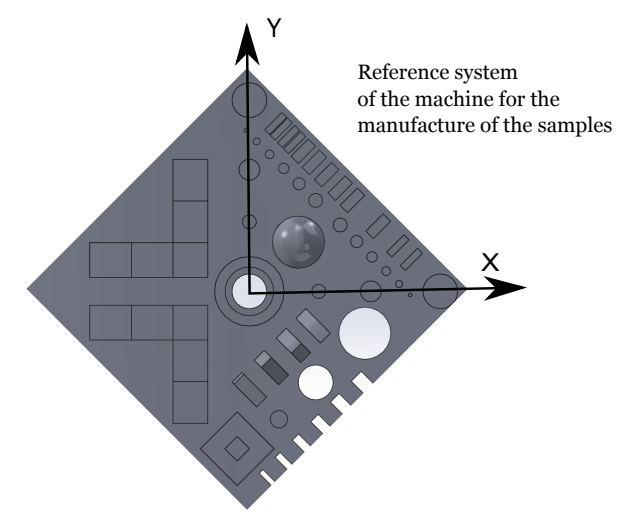

Figure 5: Coordinate system used in the manufacturing of the samples

The total time consumed by the machine was approximately 60 hours. Figure 6 shows the time for each sample.

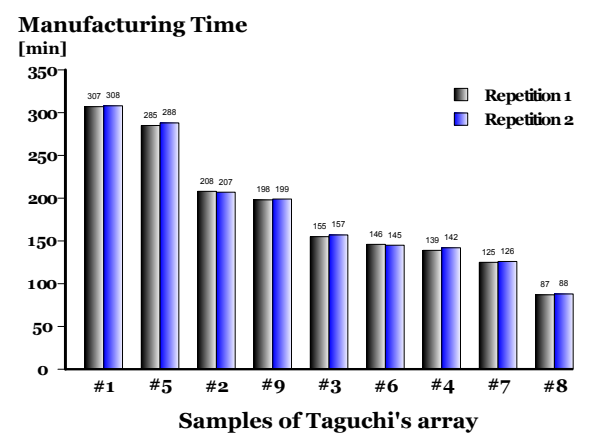

Figure 6: Fabrication time of the control factor sorted in descending order

The results show that control factors $\# 1$ (Layer thickness $=0.13 \mathrm{~mm}$, Raster width $=0.54 \mathrm{~mm}$ and Nozzle speed $=25 \mathrm{~mm} / \mathrm{s}$ ) are the most time-consuming during fabrication. By contrast, control factors \#8 
[Pre-print version], please cite as: Cruz Sanchez, F. A., Boudaoud, H., Muller, L., \& Camargo, M. (2014). Towards a standard experimental protocol for open source additive manufacturing. Virtual and Physical Prototyping, 9(3), 151-167

(Layer thickness $=0.25 \mathrm{~mm}$, Raster width $=0.62 \mathrm{~mm}$ and Nozzle speed $=75 \mathrm{~mm} / \mathrm{s}$ ) were systematically the fastest. However, the samples fabricated show that the finish surface is not the same for both control factors. These parameters have a major influence on this as shown in the fig. 7

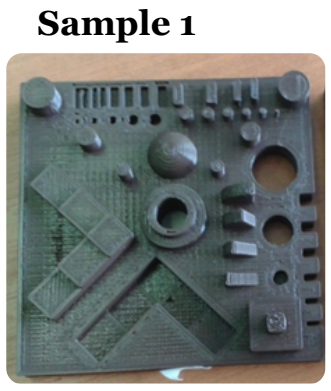

Layer Thickness $=0.13 \mathrm{~mm}$ Raster width $=0.54 \mathrm{~mm}$ Nozzle speed $=25 \mathrm{~mm} / \mathrm{s}$

\section{Sample 8}

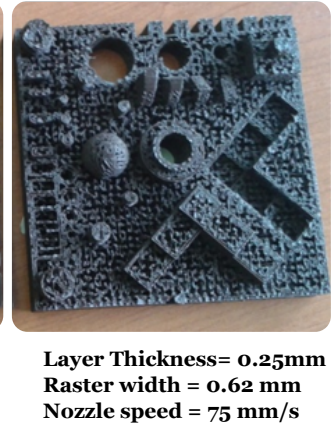

Figure 7: Surface finish of Sample 1 (slowest manufacturing time) and Sample 8 (fastest manufacturing time)

This time consumption criterion is a relevant factor in a tool for selection and characterisation of an open source 3D printer.

\section{Results and Discussion}

In this part, we will first present a statistical analysis of all the previous measurements. Then we will compare the dimensional accuracy obtained with our open source 3D printer and professional 3D printers.

As explained in the methodology (section 3), we considered three control factors (A, B, C) leading to the set of combinations shown in table 3. In order to obtain the best set of parameters regarding the geometrical accuracy, two approaches will be propose.

\subsection{Statistical Analysis}

Upon completion of the fabrication of samples, the measurements were performed using a digital Mitutoyo caliper with a measurement degree of $0.01 \mathrm{~mm}$. Each feature of the benchmarking model was measured twice, reducing the incertitude of the measurement. Therefore, a total of 3528 measurements were taken from the 18 samples fabricated. For each feature of the benchmarking model $\left(A_{i}-N_{i}\right)$, mean percent deviation value $Y 1$ for the first repetition and $Y 2$ for second repetition were found using equation (1):

$$
\% \Delta Y=\frac{\left|Y-Y_{C A D}\right|}{Y_{C A D}} 100 \%
$$

Fig. 8 shows the histogram of the database. 
[Pre-print version], please cite as: Cruz Sanchez, F. A., Boudaoud, H., Muller, L., \& Camargo, M. (2014). Towards a standard experimental protocol for open source additive manufacturing. Virtual and Physical Prototyping, 9(3), 151-167

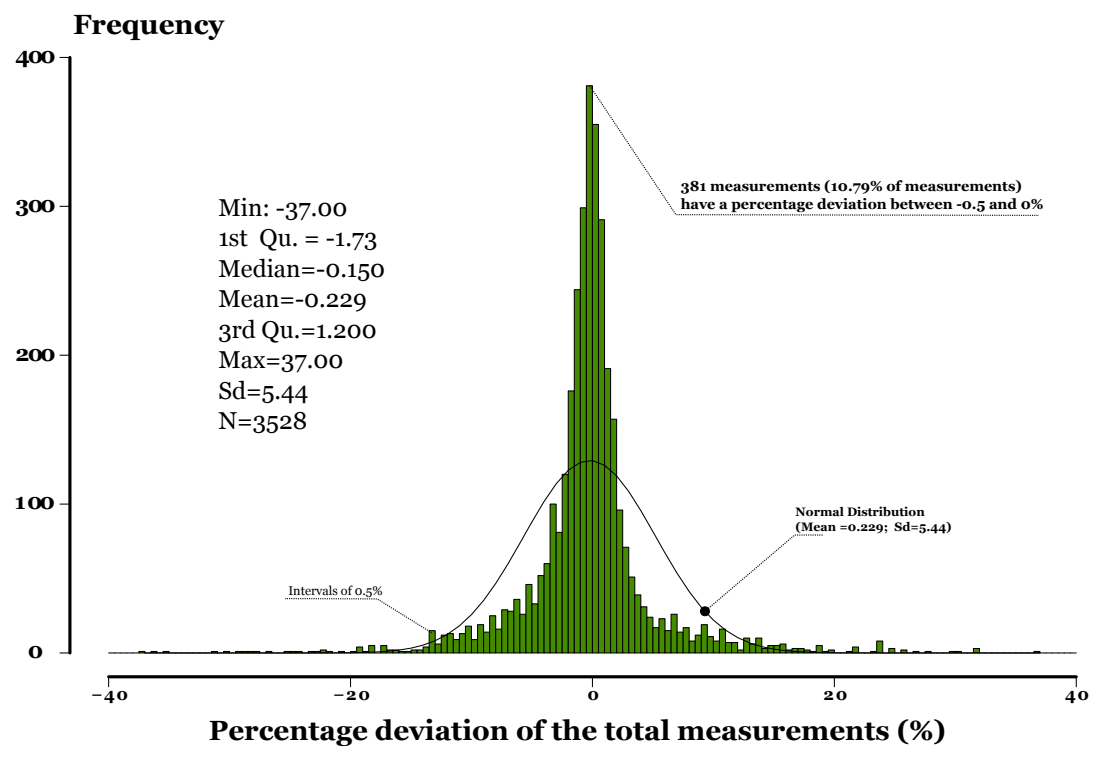

Figure 8: Distribution of the total of measurements of percent deviation

Using the classification of section 3.2, it is possible to establish the probability of the machine for making features that fall within a particular range of dimensional percentage change. This is achieved by using the probability density function for each type of dimensional accuracy proposed and for the total of data. In this way, it is feasible to compare each accuracy level in order to characterise the performance of the machine.

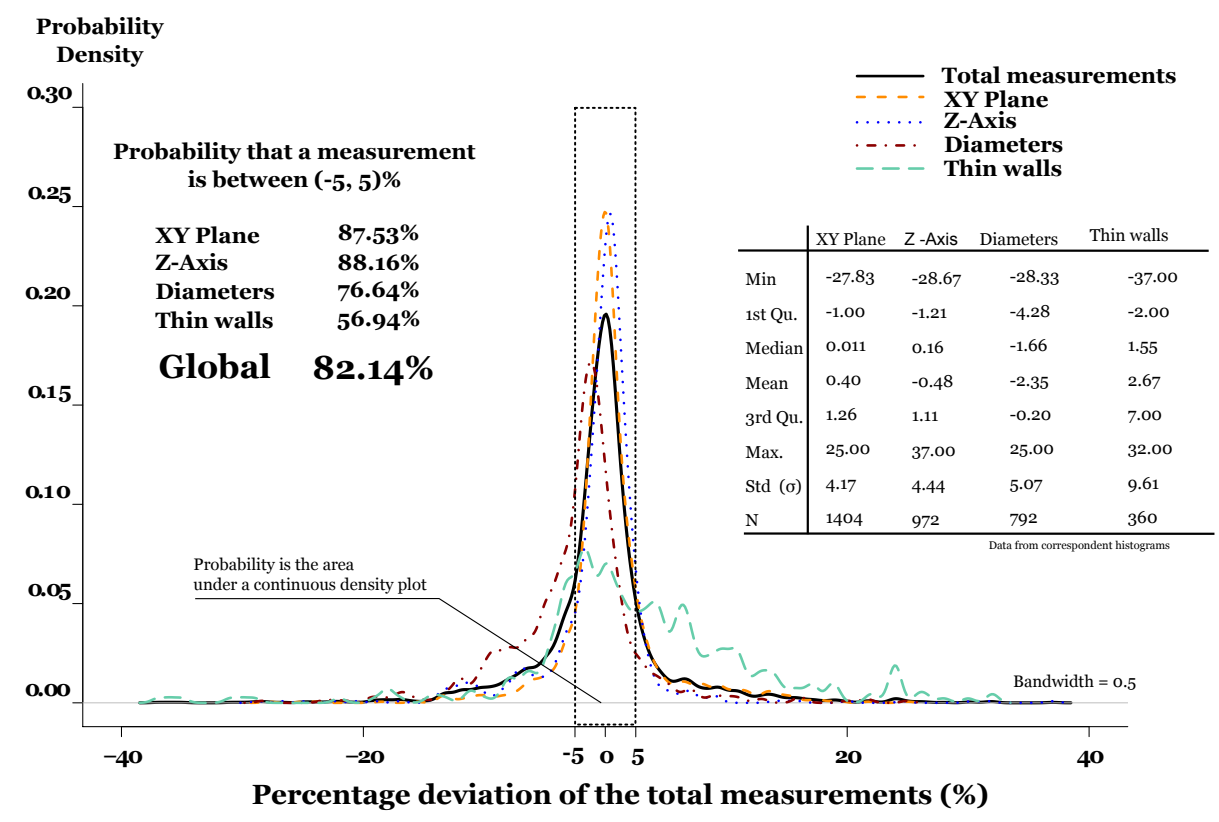

Figure 9: Density plot of the types of dimensional accuracy with the respectives probabilities values between $(-0.5 \%, 0.5 \%)$

In fig. 9, the area under the curve of each type of accuracy gives the probability of the machine for manufacturing the geometric benchmarking model within a specific range of deviation. In this research, a range of $(-5 \%, 5 \%)$ with respect to a target measurement is selected as the initial criteria. The ideal machine is one whose probability is $100 \%$ within an infinitesimal range close to zero. In this case, the results show that the overall probability of the machine is $82.14 \%$. Similarly, the probability to obtain a measurement in this range is $87.53 \%$ in the XY plane, $88.16 \%$ in the Z-Axis, $76.64 \%$ in Diameters and $56.94 \%$ in thin walls. 
[Pre-print version], please cite as: Cruz Sanchez, F. A., Boudaoud, H., Muller, L., \& Camargo, M. (2014). Towards a standard experimental protocol for open source additive manufacturing. Virtual and Physical Prototyping, 9(3), 151-167

It should be emphasised that each of those probabilities was obtained from a different quantity of data $N$. The features of the geometric benchmarking model were divided into the four types; however, this division was not equal in the number of data obtained.

\subsection{International Tolerance for the FoldaRap}

Introducing the International Tolerance (IT) grade established by ISO-ANSI standards UNI EN 20286I (1995) based on the total set of measurements taken and the corresponding deviations, the maximum tolerance grade obtained for the samples is calculated. This value places the dimensional performance of the FoldaRap machine with respect to the performance of other AM techniques. The standard tolerance value considers a tolerance factor ' $\mathrm{i}$ ' ( $\mu \mathrm{m}$ ) indicated by equation (2):

$$
i=0.45 \sqrt[3]{D}+0.001 D
$$

where $D$ is the geometric mean of the range of nominal size in $\mathrm{mm}$. In this case, the standard tolerance value is calculated for a range of nominal size. For a generic nominal dimension $D_{C A D}$, the number of tolerance unit $n$ is evaluated as follows (Singh 2011).

$$
n * i=1000\left(D_{C A D}-D_{m e a}\right)
$$

where $D_{\text {mea }}$ is the measured dimension. Using equation (3), the maximum value $n i$ among the set of measures obtained from the fabrication of the 18 samples is yielded. On a global scale, this value makes it possible to compare the performance of the open source machine 3D printer with the other AM technologies. Fig. 10 positions the results of the FoldaRap's case with regard to the work developed by Ippolito, Iuliano, \& Gatto (1995).

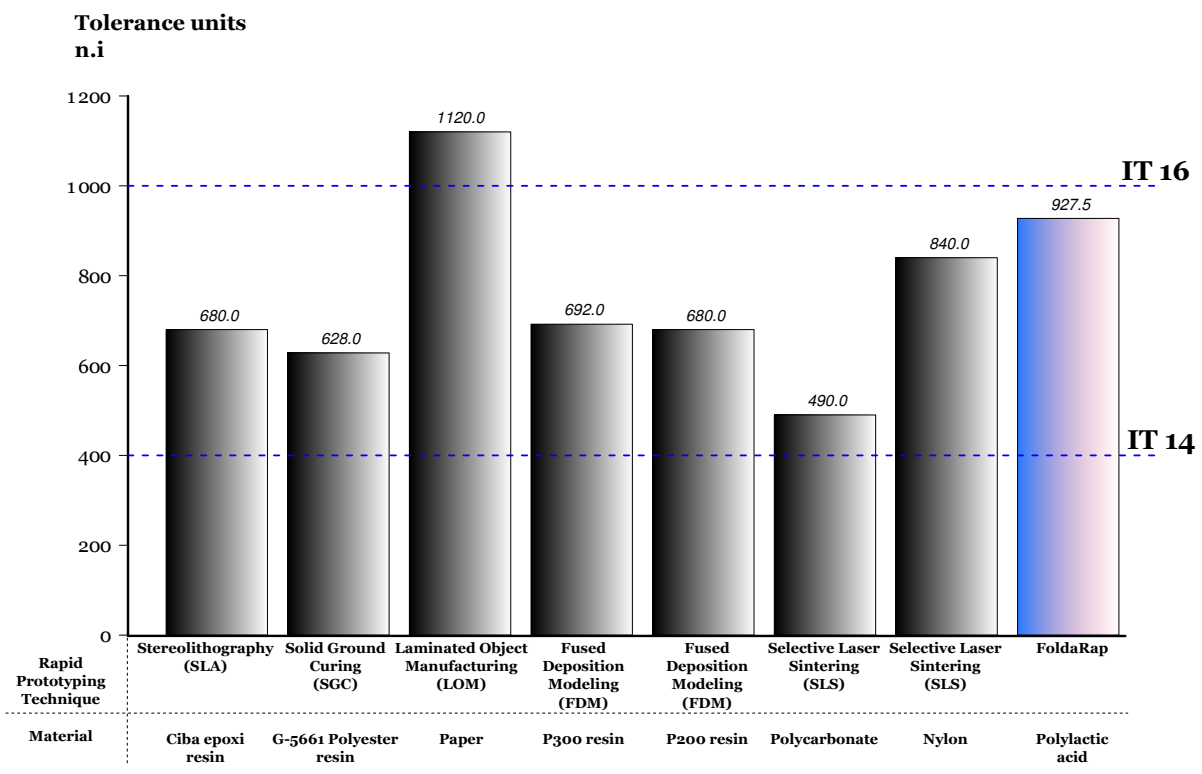

Figure 10: Comparison of the maximum tolerance grade among different AM processes

Table 6 shows the comparison of IT grades for conventional manufacturing processes, including the AM techniques (Singh 2011).

We can see through fig. 10 that, though the precision level of the FoldaRap machine is lower with respect to the different commercial AM techniques, the performance of the machine is within the range of standard tolerance grades (IT14 - IT16). This means that the tolerance of machine is from $I T 14=400 i[\mu m]$ and $I T 16=1000 i[\mu m]$, where $i$ is calculated based on equation (2). 
Table 6: Tolerance grades for various manufacturing processes

\begin{tabular}{|c|c|c|c|c|c|c|c|c|c|c|c|}
\hline & \multicolumn{11}{|c|}{ IT GRADES } \\
\hline $\begin{array}{r}\text { Process } \\
\text { Sand Casting } \\
\text { Die Casting } \\
\text { Hot Forging }\end{array}$ & 6 & 7 & 8 & 9 & 10 & 11 & 12 & 13 & 14 & 15 & 16 \\
\hline Material Removal Processes & & & & & & & & & & & \\
\hline
\end{tabular}

\subsection{Optimal control factors for the GBM}

\subsubsection{Using the DOE approach to select the most relevant control factors}

Quantifying the effect of the control factors identified in section 3.3 with the DoE method, should help to reduce the number of measurements required to evaluate the performance of the $3 \mathrm{D}$ printers. The nine experiments listed in the Taguchi table $L_{9}\left(3^{4}\right)$ gave the following results concerning the mean percent deviation value $\Delta \bar{Y}_{1}$ for the first repetition and $\Delta \bar{Y}_{2}$ for the second repetition.

\begin{tabular}{c|ccc|cc}
\multicolumn{3}{c}{ Table 7: Mean value of percent deviation } \\
\hline \# Sample & \multicolumn{3}{|c}{ Factors } & \multicolumn{2}{c}{ Reponse } \\
& A & B & C & $\Delta \bar{Y}_{1}$ & $\Delta \bar{Y}_{2}$ \\
\hline 1 & 1 & 1 & 1 & 3.307 & 2.113 \\
2 & 1 & 2 & 2 & 2.398 & 2.752 \\
3 & 1 & 3 & 3 & 2.678 & 4.213 \\
4 & 2 & 1 & 3 & 2.476 & 3.053 \\
5 & 2 & 2 & 1 & 2.328 & 3.202 \\
6 & 2 & 3 & 2 & 2.308 & 3.178 \\
7 & 3 & 1 & 2 & 2.498 & 2.930 \\
8 & 3 & 2 & 3 & 5.227 & 5.901 \\
9 & 3 & 3 & 1 & 2.935 & 3.299 \\
\hline
\end{tabular}

Calculating the mean effect of the control factors gives the following results shown in fig. 11

Table 8: Mean effect of the control factors

\begin{tabular}{l|cll}
\hline Control factors & Level & Mean value & Effect \\
\hline \multirow{3}{*}{ Layer Thickness (mm) } & 1 & 2.91 & -0.25 \\
& 2 & 2.76 & -0.40 \\
& 3 & 3.80 & 0.64 \\
\hline \multirow{3}{*}{ Raster width ( $\mathbf{m m})$} & 1 & 2.73 & -0.43 \\
& 2 & 3.63 & 0.48 \\
& 3 & 3.10 & -0.05 \\
\hline \multirow{2}{*}{ Nozzle speed (mm/s) } & 1 & 2.86 & -0.29 \\
& 2 & 2.68 & -0.48 \\
& 3 & 3.92 & 0.77 \\
\hline
\end{tabular}


[Pre-print version], please cite as: Cruz Sanchez, F. A., Boudaoud, H., Muller, L., \& Camargo, M. (2014). Towards a standard experimental protocol for open source additive manufacturing. Virtual and Physical Prototyping, 9(3), 151-167

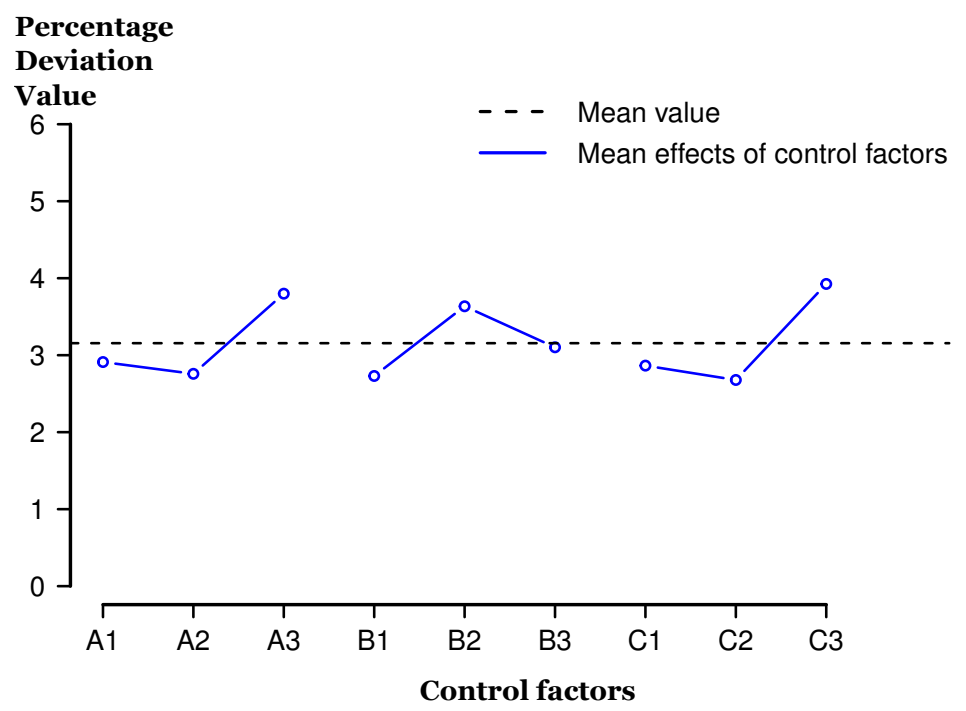

Figure 11: Variation of the percent deviation value depending on the control factors

At first glance, there is no overriding effect of control factors and it does not seem possible to reduce the model of the accuracy of the 3D printers. In order to provide a correct interpretation of the effect of factors, an ANOVA analysis is necessary. This provides significance rating of the relative influence of each factor analysed in this study. Factors which significantly influence the percent deviation of the model can be identified. A variable possessing the maximum value of variance is said to have the most significant effect on the experiment. Therefore, based on the responses of the means percentage deviation values $\Delta Y 1$ and $\Delta Y 2$ showed in Table 7, the ANOVA computations were carried out.

Table 9: ANOVA analysis

\begin{tabular}{ll|cccccc}
\hline \multicolumn{1}{l}{ Factors } & DOF & SS & V & F value & $\operatorname{Pr}(>\mathbf{F})$ & Contribution \\
\hline \multirow{4}{*}{ Y1 } & Layer thickness $[\mathrm{mm}]$ & 2 & 3.791 & 1.895 & 3.629 & 0.061 & $21.7 \%$ \\
Raster width $[\mathrm{mm}]$ & 2 & 2.483 & 1.241 & 2.377 & 0.138 & $14.2 \%$ \\
& Nozzle speed $[\mathrm{mm} / \mathrm{s}]$ & 2 & 5.430 & 2.715 & 5.198 & 0.025 & $31.1 \%$ \\
& Residuals & 11 & 5.746 & 0.522 & & & $32.9 \%$ \\
Total & 17 & 17.450 & & & & \\
\hline
\end{tabular}

ANOVA results (Table 9) show that the only significant control factor is the Nozzle speed, but more importantly, that there are unusually large residuals (contribution: 32.9\%). This confirms that the noise produced by this type of machine has more impact than the effect of the control factors. Elements of noise that can affect the experimentation are shown in fig 12.

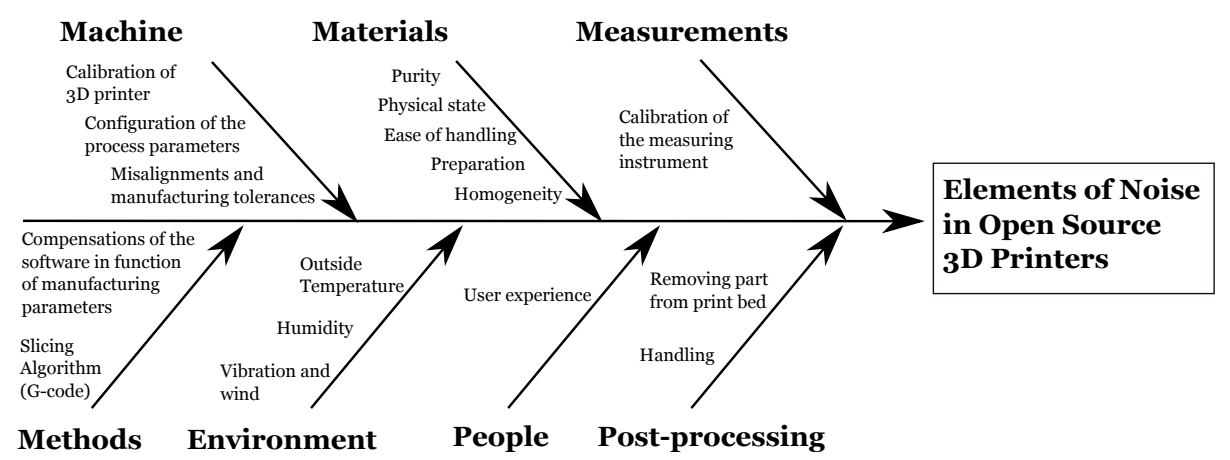

Figure 12: Ishikawa diagram of the factors that could affect in the open source 3D printers 
[Pre-print version], please cite as: Cruz Sanchez, F. A., Boudaoud, H., Muller, L., \& Camargo, M. (2014). Towards a standard experimental protocol for open source additive manufacturing. Virtual and Physical Prototyping, 9(3), 151-167

The DoE approach is therefore not relevant for machines with this precision level. Consequently, another methodology to obtain the best combination of control factors for fabrication of the GBM is proposed.

\subsubsection{Proposed method}

The objective is to rank the control factors according to Taguchi's array proposed in section 3.3, in order to find within the nine combinations those control factors with the highest accuracy. As an estimator parameter, the root-mean-square deviation (RMSD) is used. This was calculated as follows:

$$
R M S D_{i}=\sqrt{\left(\sum_{i=1}^{m} \frac{x_{i}-\bar{x}}{m}\right)^{2}+\left(\frac{\sum_{i=1}^{m} x_{i}}{m}-Y_{C A D}\right)^{2}}
$$

where the first term is the variance $\left(\sigma^{2}\right)$ of the measurements obtained and the second term is the deviation of the mean value $\bar{Y}$ with respect to the target value $\left(Y_{C A D}\right)$. The $m$ value is the quantity of measurements taken for each feature. If the RMSD value of a feature is zero, this would mean that every measurement taken is always the same $\left(\sigma^{2}=0\right)$ and this measurement is always equal to the corresponding CAD model measurement $(\bar{x}=0)$, thus, the lower the RMSD value, the more accurate the feature. Using this approach, the RMSD value of each feature gives an estimation of the machine's performance when it uses a control factor of the Taguchi array. In that way, this RMSD measurement makes it possible to rank, from best to worst, every control factor for every feature of each manufactured sample.

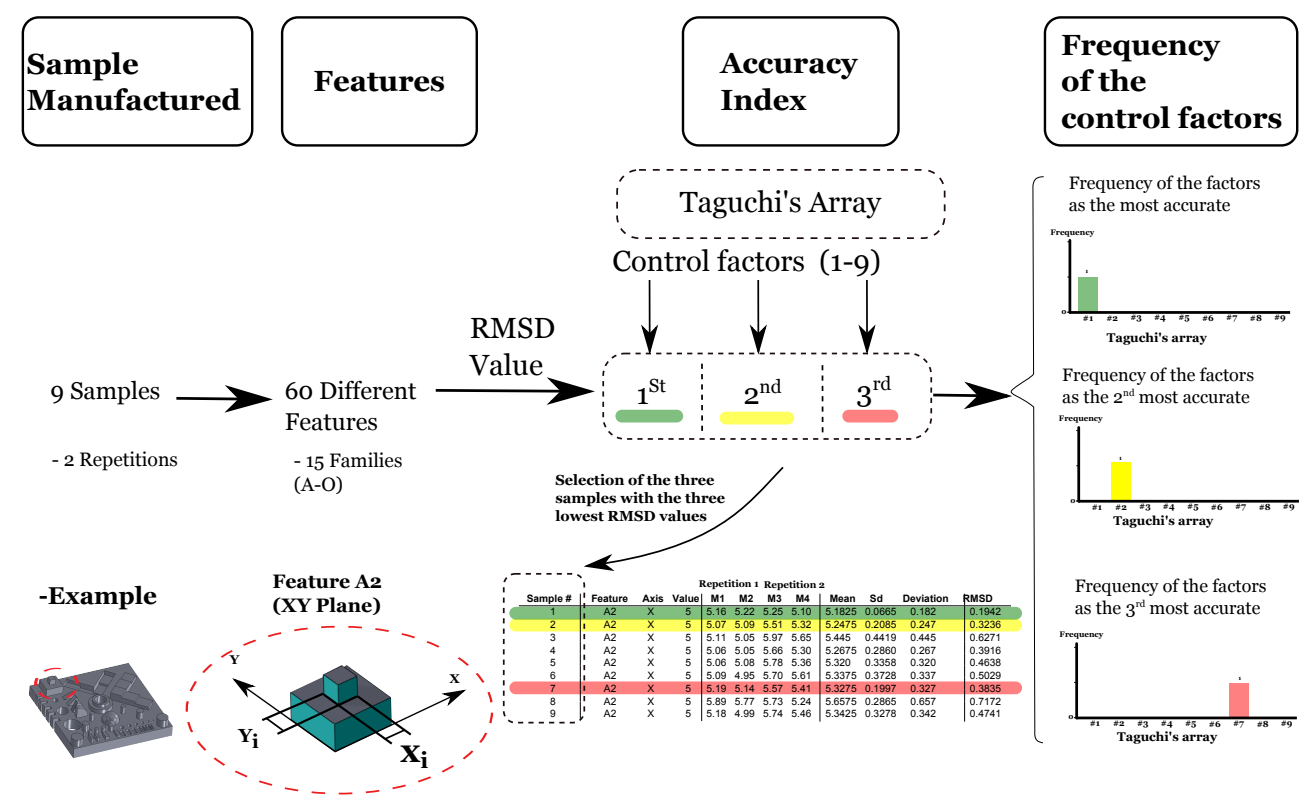

Figure 13: Methodology of selection of the three best control factors

It is intended to collect in a database the three control factors with the minimum value of RMSD for each feature. Fig. 13 schematically shows the proposed strategy. The interest of this strategy is to obtain the frequency of every control factor when they are selected as the most accurate, the second most accurate and the third most accurate. Using this frequency, we can establish a link between the performance of the 3D printer in terms of dimensional precision and the process parameters of the process. Fig. 14 shows the histograms of the control factors for each type of frequency. 

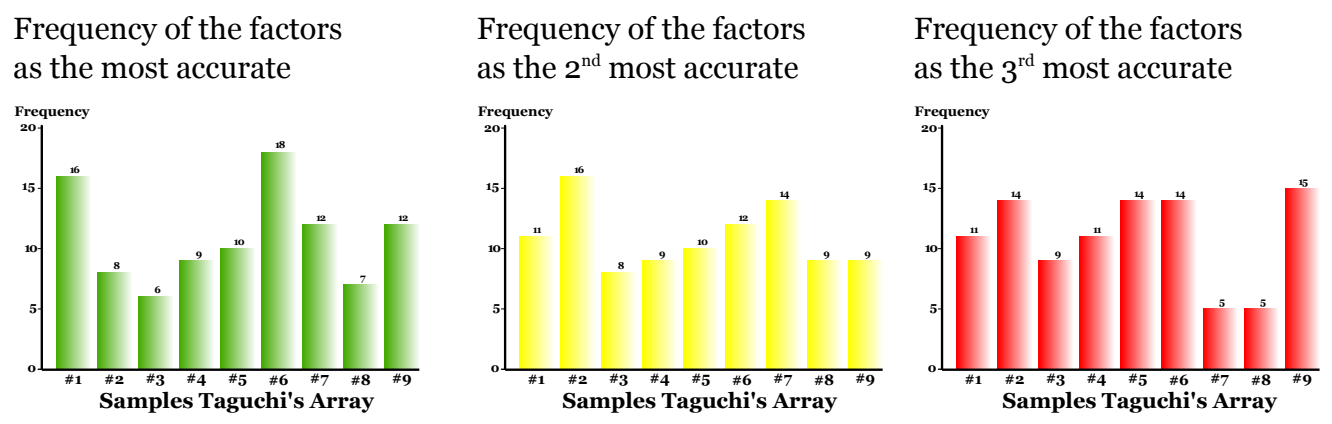

Figure 14: Frequencies of the control factors

Through the use of these frequencies, the purpose is to determine a hierarchy of the control factors in order to find out which of them has systematically been the most accurate throughout the set of measurements of the benchmarking model. As a result, a Global Index of performance is established with three relative weights $w_{i}$ for pondering the significance of each type of frequency, giving more importance to a set of frequencies of the factors qualified as the most accurate.

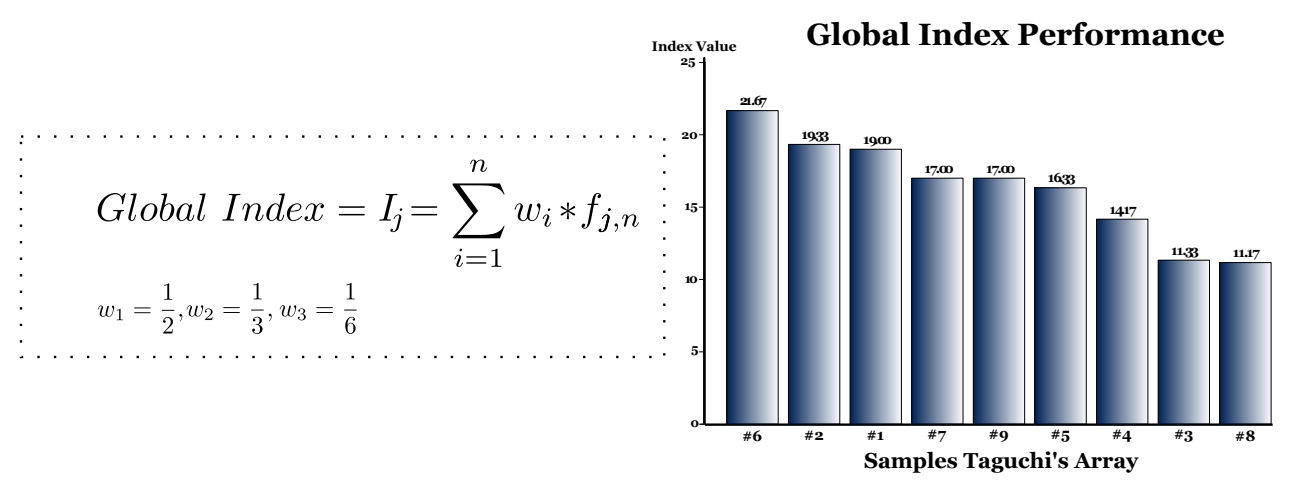

Figure 15: Performance index of each control factor tested according to Taguchi's array

Fig.15 shows the results of this approach. Taguchi's control parameters \#6 (Layer thickness $=0.18 \mathrm{~mm}$, Raster width $=0.71 \mathrm{~mm}$ and Nozzle speed movement $=50 \mathrm{~mm} / \mathrm{s}$ ) were identified as the best parameters within the total of parameters established through the use of Taguchi array.

\subsection{Discussion}

The first comment can be made with regard to build time needed for the 18 samples. In fact, it has taken nearly 60 hours to print all the samples. In order to reduce this time, it will be interesting to simplify the GBM. In fig. 16, the RSMD value is calculated for every feature (A-O) of each sample table 1. In this figure, dimensional accuracy in the XY plane and the Z-Axis for the lateral features are those having the lowest geometrical performance. Further experimentation is then needed in order to determinate whether this weakness is specific to the FoldaRap 3D printer or if it could be generalised to the Cartesian family of printers. 
[Pre-print version], please cite as: Cruz Sanchez, F. A., Boudaoud, H., Muller, L., \& Camargo, M. (2014). Towards a standard experimental protocol for open source additive manufacturing. Virtual and Physical Prototyping, 9(3), 151-167
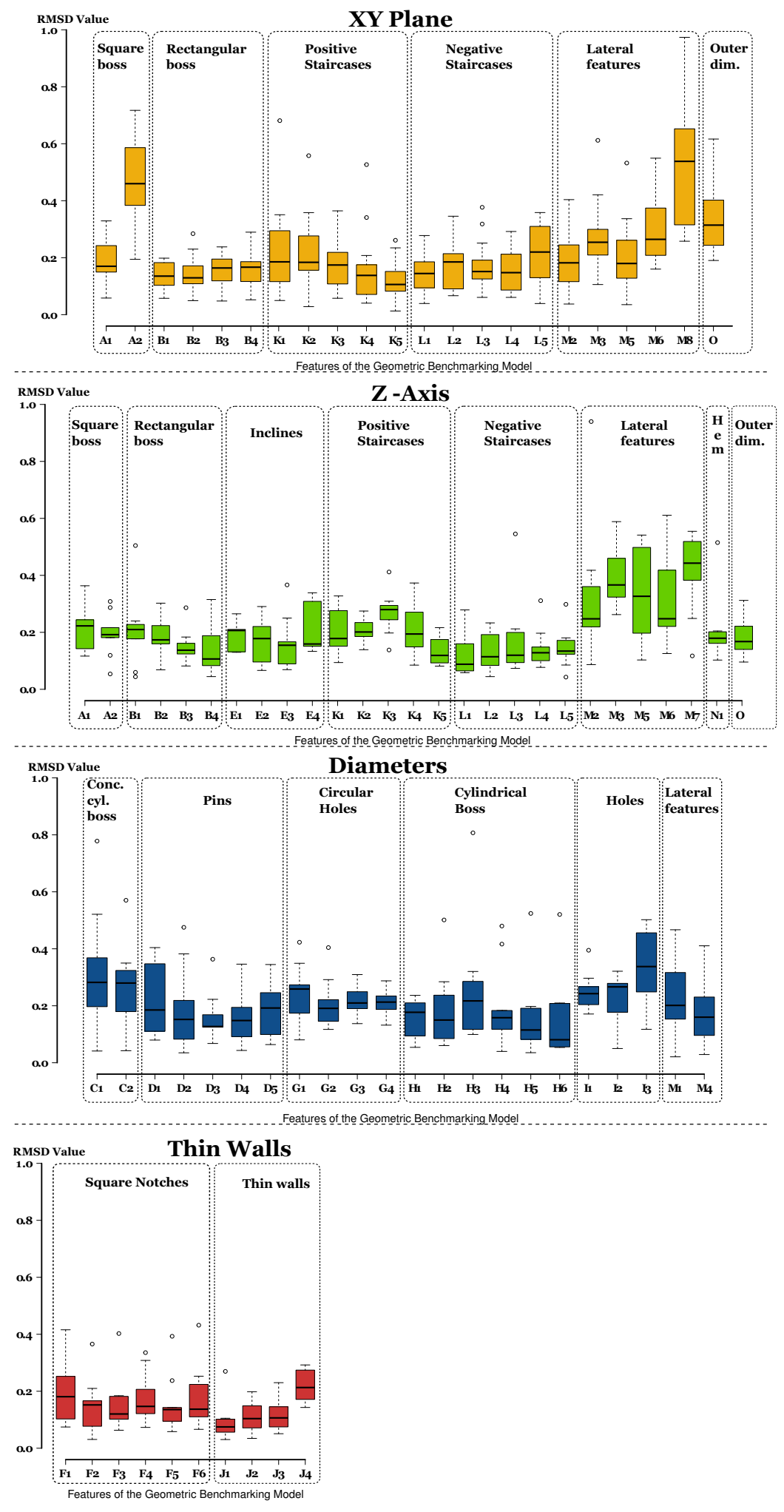

Figure 16: Variation of the RSMD value of each feature of the GBM according to the four types of accuracy

\section{Conclusion}

In the present work, an experimental protocol to evaluate open source 3D printers regarding dimensional accuracy has been proposed. We use the proposed protocol on a representative 3D printer: the FoldaRap. It was found that the International Standard Tolerance Grade of this machine is situated between IT14 and 
[Pre-print version], please cite as: Cruz Sanchez, F. A., Boudaoud, H., Muller, L., \& Camargo, M. (2014). Towards a standard experimental protocol for open source additive manufacturing. Virtual and Physical Prototyping, 9(3), 151-167

IT16.

On the other hand, the process parameters that give the highest accuracy for the fabrication of the geometric benchmarking model have been obtained using the Taguchi approach coupled with the root-meansquare deviation (RMSD) value as an accuracy estimator. The results of this approach show that the following parameters: Layer thickness $=0.18 \mathrm{~mm}$, Raster width $=0.71 \mathrm{~mm}$ and Nozzle speed movement $=$ $50 \mathrm{~mm} / \mathrm{s}$ have been systematically present with a low RMSD value in the fabrication of the 18 samples of the geometrical benchmarking model.

Furthermore, results regarding deviation of the features used suggest that the proposed protocol could be optimised in order to reduce the overall experimental time (60 hours).

\section{References}

Anitha, R, S Arunachalam, \& P Radhakrishnan (Dec. 2001). "Critical parameters influencing the quality of prototypes in fused deposition modelling". In: Journal of Materials Processing Technology 118.1-3, pp. 385-388.

Azadeh, Ali et al. (Aug. 2011). "Design and implementation of an integrated Taguchi method for continuous assessment and improvement of manufacturing systems". In: The International Journal of Advanced Manufacturing Technology 59.9-12, pp. 1073-1089.

Bayless, Jacob, Mo Chen, \& Bing Dai (2010). Wire Embedding 3D Printer. Tech. rep. University of British Columbia.

Birtchnell, Thomas \& John Urry (Sept. 2013). "Fabricating Futures and the Movement of Objects". In: Mobilities 8.3, pp. 388-405.

Campbell, Thomas et al. (2011). Could 3D Printing Change the World?. Technologies, Potential, and Implications of Additive Manufacturing. Tech. rep. Washington, DC: Atlantic Council.

Canessa, E., C. Fonda, \& M Zennaron, eds. (2013). Low-Cost 3D Printing for science, Education Sustainable development. Trieste: ICTP - The Abdus Salam International Centre for Theroretical Physics.

Cantini, Lorenzo \& Ivan Bortolin (2012). Slic3r User Guide. Tech. rep.

Ciurana, Joaquim de, Lídia Serenóa, \& Ėlia Vallès (Jan. 2013). "Selecting Process Parameters in RepRap Additive Manufacturing System for PLA Scaffolds Manufacture". In: Procedia CIRP 5, pp. 152-157.

Crump, S.S. (June 1988). Apparatus and method for creating three-dimensional objects.

Economist, The (2012). "A third industrial revolution: special report: Manufacturing and innovations". In: The Economist.

Evans, Brian (2012). Practical 3D Printers: The Science and Art of 3D Printing. New York, NJ: Apress, p. 321.

Fahad, Muhammad \& Neil Hopkinson (2012). "A new benchmarking part for evaluating the accuracy and repeatability of Additive Manufacturing processes". In: 2nd International Conference on Mechanical, Production and Automobile Engineering (ICMPAE'2012). Singapore, pp. 234-238.

Fowlkes, William Y. \& Clyde M. Creveling (1995). Engineering Methods for Robust Product Design: Using Taguchi Methods in Technology and Product Development. Reading, MA: Addison Wesley Longman, Inc., p. 432.

Garrido, Paulo (Aug. 2010). "Open design and knowledge integration in semiotic manufacturing integration". In: International Journal of Computer Integrated Manufacturing 23.8-9, pp. 819-831.

Giacalone, Jean-Marc (2014). EMAKER Huxley - RepRapWiki.

Gilloz, Emmanuel (2011a). Pocket Laser Engraver.

- (2011b). VertX.

- (2014). FoldaRap - RepRapWiki.

Holland, D, O'Donnell, G., Bennett, G. (2010). "Open Design and the RepRap Project”. In: 27th International Manufacturing Conference. Galway, Ireland, pp. $97-106$.

Ippolito, R, L Iuliano, \& A Gatto (Jan. 1995). "Benchmarking of Rapid Prototyping Techniques in Terms of Dimensional Accuracy and Surface Finish". In: CIRP Annals - Manufacturing Technology 44.1, pp. 157160 . 
[Pre-print version], please cite as: Cruz Sanchez, F. A., Boudaoud, H., Muller, L., \& Camargo, M. (2014). Towards a standard experimental protocol for open source additive manufacturing. Virtual and Physical Prototyping, 9(3), 151-167

Jayaram, Dureen et al. (1994). "Benchmarking of Rapid Prototyping Systems - Beginning to Set Standards". In: Solid Freeform Fabrication Proceedings. Ed. by Marcus Harris L. et al. Austin, pp. 146-153.

Johnson, W. M. et al. (2011). "Benchmarking evaluation of an Open Source Fused Deposition Modeling Additive Manufacturing System". In: Proc of the 22nd Annual International Solid Freeform Fabrication Symposium. Austin, Texas, pp. 197-211.

Jones, Rhys et al. (Jan. 2011). "RepRap - the replicating rapid prototyper". In: Robotica 29.01, pp. 177-191.

Kostakis, Vasilis \& Marios Papachristou (Aug. 2014). "Commons-based peer production and digital fabrication: The case of a RepRap-based, Lego-built 3D printing-milling machine". In: Telematics and Informatics 31.3, pp. 434-443.

Kruth, J.P. (1991). "Material Incress Manufacturing by Rapid Prototyping Techniques". In: CIRP Annals Manufacturing Technology 40.2, pp. 603-614.

Kumar Sahu, Ranjeet (2011). "An Investigation on Dimensional accuracy of Fused Deposition Modelling (FDM) processed part using Fuzzy logic". PhD thesis. National Institure of Technology Rourkela Orissa.

Leigh, Simon J et al. (Jan. 2012). "A simple, low-cost conductive composite material for 3D printing of electronic sensors." In: PloS one 7.11, e49365.

Mahesh, M. (2004). "Rapid Prototyping and Manufacturing Benchmarking". PhD thesis. National University of Singapore.

Mahesh, M. et al. (Jan. 2006). "A Six-sigma approach for benchmarking of RP\&M processes". In: The International Journal of Advanced Manufacturing Technology 31.3-4, pp. 374-387.

MakerBot (2014). MakerBot Industries.

Malone, Evan \& Hod Lipson (2007). "Fab@Home: the personal desktop fabricator kit". In: Rapid Prototyping Journal 13.4, pp. 245-255.

Moylan, Shawn et al. (2012). "Proposal for a standardized test artifact for additive manufacturing machines and processes". In: Proceedings of the Solid Freeform Fabrication Symposium, pp. 902-920.

Pearce, J .M. et al. (2010). "3-D Printing of Open Source Appropriate Technologies for Self-Directed Sustainable Development". In: Journal of Sustainable Development 3.4, pp. 17-29.

Pearce, Joshua M (Sept. 2012). "Materials science. Building research equipment with free, open-source hardware." In: Science (New York, N.Y.) 337.6100, pp. 1303-4.

Pearce, Joshua M. (2014). Open-Source Lab. Elsevier, pp. 95-162.

Perez, Mireya A et al. (2013). "Ranking Model For 3D Printers". In: International Solid Freeform Fabrication Symposium An Additive Manufacturing Conference. Austin, Texas, USA, pp. 1048-1065.

RepRap.org (2014). RepRap Family Tree - RepRapWiki.

RepRapPro (2014). Melzi - RepRapWiki.

RepRapWiki (2014). RepRap Options -.

Rifkin, Jeremy (2012). The Third Industrial Revolution: How Lateral Power Is Transforming Energy, the Economy, and the World. Palgrave Macmillan.

Roberson, D.a., D. Espalin, \& R.B. Wicker (Sept. 2013). "3D printer selection: A decision-making evaluation and ranking model". In: Virtual and Physical Prototyping 8.3, pp. 201-212.

Scaravetti, Dominique, Patrice Dubois, \& Robert Duchamp (Oct. 2007). "Qualification of rapid prototyping tools: proposition of a procedure and a test part". In: The International Journal of Advanced Manufacturing Technology 38.7-8, pp. 683-690.

Sells, Ed et al. (2007). "RepRap: The replicating Rapid Prototyper Maximizing Customizability by Breeding the Means of Production". In: The $200^{7}$ World Conference on Mass Customization and Personalization. $M C P C$ 200\%. Cambridge, MA.

Singh, Rupinder (June 2011). "Process capability study of polyjet printing for plastic components". In: Journal of Mechanical Science and Technology 25.4, pp. 1011-1015.

Sood, Anoop Kumar, R.K. Ohdar, \& S.S. Mahapatra (Dec. 2009). "Improving dimensional accuracy of Fused Deposition Modelling processed part using grey Taguchi method". In: Materials 63 Design 30.10, pp. 42434252.

Ter, Chap (2014). RepRap for Science - How to Use, Design, and Troubleshoot the Self-Replicating 3-D Printer, pp. 95-162. 
[Pre-print version], please cite as: Cruz Sanchez, F. A., Boudaoud, H., Muller, L., \& Camargo, M. (2014). Towards a standard experimental protocol for open source additive manufacturing. Virtual and Physical Prototyping, 9(3), 151-167

Xu, F, Y S Wong, \& H T Loh (2000). "Toward Generic Models for Comparative Rapid Prototyping and Manufacturing". In: Journal of Manufacturing Systems 19.5, pp. 283-296.

Zhang, Chenlong et al. (Jan. 2013). "Open-source 3D-printable optics equipment." In: PloS one 8.3, e59840. Zhou, Jack G., Daniel Herscovici, \& Calvin C. Chen (Feb. 2000). "Parametric process optimization to improve the accuracy of rapid prototyped stereolithography parts". In: International Journal of Machine Tools and Manufacture 40.3, pp. 363-379.

\section{Notations}

AM Additive Manufacturing

DLS Direct Laser Sintering

FDM Fused Deposition Modeling

GBM Geometric Benchmarking Model

LOM Laminated Object Manufacturing

SGC Solid Ground Curing

SL Stereolithography

SLS Selective Laser Sintering 